Canadian University Music Review

Canadian University Music Review

Revue de musique des universités canadiennes

\title{
Une œuvre et son titre : à propos du Carnaval, op. 9, de Robert Schumann
}

\section{Pierre Vachon}

Volume 18, numéro 2, 1998

URI : https://id.erudit.org/iderudit/1014651ar

DOI : https://doi.org/10.7202/1014651ar

Aller au sommaire du numéro

\section{Éditeur(s)}

Canadian University Music Society / Société de musique des universités canadiennes

ISSN

0710-0353 (imprimé)

2291-2436 (numérique)

Découvrir la revue

Citer cet article

Vachon, P. (1998). Une œuvre et son titre : à propos du Carnaval, op. 9, de Robert Schumann. Canadian University Music Review / Revue de musique des universités canadiennes, 18(2), 1-24. https://doi.org/10.7202/1014651ar
Résumé de l'article

Qu'y a-t-il dans le titre d'une œuvre musicale qui permettrait de mieux comprendre l'œuvre elle-même, sa genèse, sa construction, sa signification et le symbolisme dont elle pourrait se réclamer? Au-delà d'une constellation de fragments, le Carnaval, op. 9, de Robert Schumann porte en lui une logique motivique et symbolique que l'étude du titre permet de démasquer. L'auteur tente de montrer qu'une étude titrologique mettra au jour les liens très intimes entre musical et extramusical ainsi que le réseau de référents très complexe vers lequel le titre dirige. Le titre agit bien souvent comme interface entre l'œuvre musicale et son symbolisme.
All Rights Reserved ( C Canadian University Music Society / Société de musique des universités canadiennes, 1998
Ce document est protégé par la loi sur le droit d'auteur. L’utilisation des services d'Érudit (y compris la reproduction) est assujettie à sa politique d'utilisation que vous pouvez consulter en ligne.

https://apropos.erudit.org/fr/usagers/politique-dutilisation/ 


\title{
UNE GUVRE ET SON TITRE : À PROPOS DU CARNAVAL, OP. 9, DE ROBERT SCHUMANN
}

\author{
Pierre Vachon
}

Le Carnaval, op. 9, pour piano (1834-35) de Robert Schumann est un cycle composé de 21 miniatures ou "pièces caractéristiques », genre cultivé dans le cadre de la musique à programme au $\mathrm{XIX}^{\mathrm{e}}$ siècle. En tant que telles, les pièces caractéristiques ont ceci de particulier qu'elles sont porteuses de titres à teneur souvent poétique. Si, à travers l'histoire de la musique, on se surprend à trouver des intitulés surtout imitatifs, descriptifs et génériques - Le caquet des femmes ou La guerre de Janequin, telle fugue de Bach, telle sonate de Mozart ou encore telle symphonie de Haydn - , le romantisme semble redéfinir la nature de l'intitulé. Ainsi, les œuvres caractéristiques du romantisme portent des titres " conditionnés par la peinture ou la poésie (Aquarelle, Humoresque), [les titres] deviennent progressivement plus fantaisistes, plus subjectifs, plus hermétiques. [...] ils sont souvent pleins de sous-entendus ou vont même jusqu'à laisser à l'auditeur le soin d'interpréter le sens de la pièce ${ }^{1}$. » Est-ce à dire que les pièces caractéristiques pourraient alors relever d'une association esthétique entre musical et extramusical, le titre agissant comme interface entre la musique et sa signification?

Le présent article porte précisément sur le titre de l'œuvre musicale; il cherche à mettre en lumière certains liens que l'intitulé entretient avec l'œuvre, de la genèse de celle-ci au symbolisme de l'intitulé. En prenant appui principalement sur les écrits de Schumann - Tagebücher ${ }^{2}$, Gesammelte Schriften über Musik und Musiker ${ }^{3}$, articles de la Neue Zeitschrift für Musik ${ }^{4}$, correspondance $^{5}$-, je me propose un examen sommaire du titre de son Carnaval qui montrera comment le travail musical du compositeur puise à des sources extramusicales et que l'environnement même de ce dernier, des conditions de vie aux dispositions d'esprit, a une incidence sur sa création. Chez Schumann,

1 Marc Honegger, édit., Dictionnaire de la musique (Paris : Bordas, 1970-76), art. «Pièce de genre ou pièce caractéristique ".

2Robert Schumann, Tagebücher, Band I : 1827-1838, édit. par Georg Eismann (Leipzig : VEB Deutscher Verlag für Musik, 1971); Tagebücher, Band II : 1836-1854, édit. par Gerd Nauhaus (Leipzig : VEB Deutscher Verlag für Musik, 1987); Tagebücher, Band III, Haushaltbücher, parties 1 (1837-1847) et 2 (1847-1856), édit. par Gerd Nauhaus (Leipzig : VEB Deutscher Verlag für Musik, 1982).

3 Gesammelte Schriften über Musik und Musiker von Robert Schumann, $5^{\mathrm{e}}$ éd., 2 vol., édit. par Martin Kreisig (Leipzig : Breitkopf und Härtel, 1914).

4 Intégrés aux Gesammelte Schriften.

5F. Gustav Jansen, Robert Schumanns Briefe: Neue Folge, $2^{\mathrm{e}}$ éd., revue et augmentée (Leipzig : Breitkopf und Härtel, 1904). 
la musique pour piano seul des années 1830-40 est intimement liée à l'extramusical et le titre devient indissociable de l'œuvre, titre et œuvre s'éclairant mutuellement.

\section{La titrologie : une approche}

L'étude du titre, de son histoire et de ses fonctions constitue le champ d'investigation de la " titrologie », discipline vouée principalement à l'étude du paratexte littéraire ${ }^{6}$. Plus récemment, la titrologie a été appliquée aux arts plastiques et à la musique afin d'éclairer la proximité qui existe entre le titre et l'œuvre, proximité peut-être plus grande qu'on ne l'imagine, et qui fait croire à une sorte de complicité signifiante et symbiotique. A priori, le titre peut sembler peu éloquent, voire banal, en regard de l'œuvre, mais peut-être est-il, a posteriori, beaucoup plus révélateur qu'il n'y paraît, agissant comme témoin privilégié de tendances esthétiques, de pratiques éditoriales et de modes sociales. On aurait sans doute tort de sous-estimer l'apport de cet élément, car "à observer le seul phénomène de désintitulation et de réintitulation des mêmes œuvres musicales au cours du temps, [on lit] en creux l'histoire de la musique $^{7}$. » À une époque, le XIX ${ }^{\mathrm{e}}$ siècle, où le sens même d'une œuvre musicale est dans certains cas tributaire d'une association littéraire, une étude titrologique d'œuvres instrumentales romantiques revêt son importance pour expliciter les pratiques d'intitulation et le symbolisme inhérent aux intitulés choisis.

À l'époque de Schumann, l'alliance du mot et de la musique confère à l'extramusical une autorité accrue en rapport avec l'œuvre musicale. Les intitulés des œuvres instrumentales se limitent de moins en moins à des formules génériques comme sonate, valse, polonaise, variation et prélude et renvoient davantage à des événements, à des personnes, à des situations, à une légende, etc., prêtant à l'œuvre un contenu non musical qui, selon les tenants de la Neudeutsche Schule (Nouvelle école allemande), conférerait une signification à l'œuvre instrumentale.

Schumann s'est longuement exprimé sur la musique à programme ou à titre, ayant lui-même recours, presque systématiquement pendant les années

6Les principaux travaux récents de titrologie sont les suivants. En littérature : (1) Leo H. Hoek, La marque du titre : dispositifs sémiotiques d'une pratique textuelle (La Haye : Mouton, 1981), une description sémiotique des marques laissées par le titre sur un texte; (2) Gérard Genette, Seuils (Paris : Éditions du Seuil, 1987), qui consiste en un essai de critique littéraire portant sur l'appareil paratextuel auquel appartient le titre; (3) Christian Moncelet, Essai sur le titre : en littérature et dans les arts (Le Cendre: Éditions BOF, 1972), qui consacre un court chapitre (p. 159-79) à la question du titre des œuvres musicales; (4) Colette Kantorowicz, « Éloquence des titres » (thèse de doctorat [linguistique], New York University, 1986), qui applique la théorie de la nouvelle rhétorique de Chaim Perelman, auteur de plusieurs travaux sur la rhétorique et l'argumentation. En musique : (1) Moncelet, Essai sur le titre; (2) Marcelle Guertin, «L'isle joyeuse : à quel titre? », Amadis 1 (1997) : 89-108, qui définit une nomenclature des titres (objectifs ou associatifs) puis dresse une grille de caractérisation; (3) Françoise Escal, Aléas de l'auvre musicale, collection Savoir : cultures (Paris : Hermann, 1996), qui, en retraçant l'histoire des titres des œuvres musicales, décrit les pratiques et les messages qui accompagnent l'œuvre musicale et montre comment ces signes constituent une zone de transaction entre l'œuvre et ses destinataires et qu'ils infléchissent la réception de ces œuvres.

7Escal, Aléas de l'œuvre musicale, 294-95. 
1830-40, à des titres évocateurs pour ses œuvres instrumentales dédiées au piano : Papillons, Nachtstücke, Carnaval, Kreisleriana, Waldscenen, Arabeske, Humoreske, Fantasie, Novelletten. À titre de critique musical, il a suivi de près les créations de son époque, témoignant notamment de l'émergence de programmes, d'épigraphes et de titres dans les œuvres instrumentales. Dans son texte le plus célèbre, sa critique de la Symphonie fantastique de Berlioz publiée en 1835 dans la Neue Zeitschrift für Musik, Schumann livre certaines de ses idées maîtresses sur le titre, son importance et son apport.

Les cinq titres principaux auraient suffi; les autres détails de ces événements auraient été transmis assez rapidement par tradition orale. L'intérêt éveillé pour la personne du compositeur, qui vit à travers la symphonie elle-même, aurait garanti cela ${ }^{8}$.

Schumann ne s'opposait pas tant à l'emploi d'un titre, comme on l'a souvent écrit, qu'au recours à une trop grande quantité d'éléments extramusicaux (programme, explications, etc.). En adjoignant à la musique des titres et un programme détaillé, le pouvoir suggestif de l'extramusical est affaibli par l'explication qui lui est ajoutée. Dans le cas de la Symphonie fantastique, Schumann est d'avis que le seul titre des mouvements éveille une idée, engendre un climat et produit une émotion que la musique soutient par son effet. Ainsi, quelques mots suffisent pour provoquer l'imaginaire (c'est du moins ce que croyait Schumann), alors que la description l'endigue, et le titre fait fonction de catalyseur dans l'appréciation et la compréhension de l'œuvre. Cette position esthétique de Schumann se reflète dans de nombreux autres articles sur la musique, comme le montre un passage relatif à une étude d'Adolf Henselt intitulée Ave Maria :

Voici un exemple qui illustre comment un titre bien choisi peut accroître l'effet de la musique. Sans ce titre, la plupart exécuteraient l'œuvre comme une étude de Cramer?.

Certains spécialistes de Schumann comme Thomas Alan Brown ${ }^{10}$ et Leon Plantinga ${ }^{11}$ adhèrent entièrement au principe schumannien que la musique produit un effet. Ils souscrivent en plus à cet autre principe schumannien voulant que la musique traduise des dispositions de l'esprit ou des états d'âme (Stimmungen ${ }^{12}$ ). Sous cet angle, si l'œuvre est la manifestation audible d'une

8 « Jedenfalls hätten die fünf Hauptüberschriften genügt; die genaueren Umstände, die allerdings der Person des Componisten halber, der die Sinfonie selbst durchlebt, interessiren müssen, würden sich schon durch mündlicher Tradition fortgepflanzt haben. » Gesammelte Schriften, 1:83.

9 « Hier ist das Beispiel, wie eine gutgewählte Überschrift dir Wirkung der Musik hebt. Ohne jene Überschrift würde es von den meisten wie eine Etüde von Cramer abgespielt worden sein ». Gesammelte Schriften, 1:390.

10Thomas Alan Brown, The Aesthetics of Robert Schumann (Westport, Conn. : Greenwood Press Publishers, 1975).

11 Leon Plantinga, Schumann as Critic (New York : Da Capo Press, 1976).

12Stimmung (plur. Stimmungen) se traduit par disposition ou état de l'esprit et revient comme un leitmotiv dans le langage écrit de Schumann. Pour Schumann, le compositeur se trouve dans une 
disposition de l'esprit, l'extramusical pourrait circonscrire l'aire herméneutique propre à l'œuvre, ou à tout le moins s'en approcher, et réduire la distance entre l'œuvre et sa signification. Le titre fait donc office de clé herméneutique et s'inscrit dans un processus, l'intitulation, lui-même l'une des étapes du processus de gestation de l'œuvre. Le titre est alors partie prenante et prégnante dans le processus de composition; il s'associe à l'œuvre pour tenter d'en communiquer son essence.

L'emploi de tels titres pour les compositions musicales, usage qui est redevenu une habitude récemment, est de temps en temps critiqué. On dit qu'une bonne pièce musicale n'a pas besoin de telles indications. Certainement pas, ce qui ne veut pas dire qu'elle perd de sa valeur si elle en a, sans oublier qu'elles constituent le moyen le plus sûr par lequel le compositeur se prémunit des équivoques autour du caractère de la composition ${ }^{13}$.

On trouve des titres génériques dans la production schumannienne, mais ils sont en minorité, à la faveur de titres poétiques, investis d'un pouvoir de suggestion et susceptibles d'éveiller l'imaginaire du compositeur, de l'interprète et de l'auditeur; leur large part dans la production pour piano de Schumann interroge inévitablement leur raison d'être, voire leur validité.

[...] les titres [...] sont nécessaires pour nous [les compositeurs] pour notre travail... Je choisis simplement un titre significatif qui a autant de validité que de signification pour l'histoire entière ${ }^{14}$.

\section{Genèse du Carnaval}

En 1834, Schumann écrit dans son livre de projets : « l'année la plus significative de ma vie " ${ }^{15}$; elle marque la fin d'une période sombre, celle de l'année 1833 pendant laquelle il est en proie à une dépression. Rétabli, Schumann commence à publier en avril 1834 la Neue Zeitschrift für Musik ${ }^{16}$, un périodique qui dénonce la musique légère et ses promoteurs. Il signe ses articles des pseudonymes Florestan et Eusebius, contreparties des personnages Vult et

disposition d'esprit donnée au moment de l'émergence de l'idée musicale. Or, la musique devient le véhicule ou l'expression de cet état. Brown, The Aesthetics of Robert Schumann, 25.

13 « Man hat diese Überschriften über Musikstücke, die sich in neuerer Zeit wieder vielfach zeigen, hier und da getadelt und gesagt, eine gute Musik bedürfe solcher Fingerzeige nicht. Gewiß nicht : aber sie büßt dadurch ebensowenig etwas von ihrem Wert ein, und der Komponist beugt dadurch offenbarem Vergreifen des Charakters am sichersten vor. " Gesammelte Schriften, 1:361.

14 « [...] Überschriften sind uns also für unser Werk nötig, und wahrlich tüchtige; [...] wähle ich sinnreich bloß einen Namen, der für die ganze Handlung schon Vollgültigkeit und Deutung hat. » Gesammelte Schriften, 1:137.

15 « Merkwürdigstes Jahr meines Lebens. » Schumann, Projektenbuch (Zwickau, Robert-Schumann-Haus, archive $\mathrm{n}^{\circ} 4871$ VII C, 8 A31), 36. Cité dans Michael Jude Luebbe, « A History of Robert Schumann's Characteristic Music for Solo Pianoforte : A Study of the Compositional Process in Sketches, Autographs, and Stichvorlagen " (thèse de doctorat, University of Cincinnati, 1995), 107.

16Dans sa Neue Zeitschrift für Musik, Schumann et un groupe d'amis compositeurs et écrivains se comparent à David et à ses compagnons de la Bible, en guerre contre la tribu des philistins : ils se nomment les Davidsbündler (Compagnons de David), une confrérie mi-réelle, mi-imaginaire. 
Walt du roman Flegeljahre de Jean Paul et représentation imaginaire de sa dualité.

C'est en décembre $1834{ }^{17}$ précisément que Schumann commence la composition du Carnaval; il l'achèvera l'été suivant. Dans cette nouvelle composition, Florestan et Eusebius sont caractérisés musicalement pour la première fois et donnent chacun leur nom à une pièce : Eusebius est rêveur, mélancolique et lyrique, tandis que Florestan est passionné, flamboyant et fougueux et «pourrait provenir du nom du personnage Fidelio dans l'opéra éponyme de Beethoven ${ }^{18}$. » Bien que Schumann termine le Carnaval à l'été 1835 , ce n'est que le 28 décembre 1836 qu'il en fait parvenir une copie à l'éditeur Schlesinger à Paris pour faire publier sa nouvelle composition; rien n'en sortira. Il soumet la même demande à Breitkopf und Härtel :

Je saisis l'occasion pour vous demander, comme j'avais l'intention depuis longtemps de le faire, de vous occuper de la publication de deux de mes compositions. L'une s'intitule «Carnaval » et l'autre «Phantasien [Phantasiestücke] pour pianoforte ». Carnaval sera publié en même temps par Schlesinger à Paris, mais modifié en une forme quelque peu différente, plus adéquate pour le public parisien, et je vous demanderais de mentionner sa compagnie sur la page de titre ${ }^{19}$.

Le 31 mai 1837, Schumann redemande la partition à Breitkopf afin de lui apporter les dernières modifications; il en éliminera certains numéros dans l'espoir que le cycle soit publié en un seul recueil, accentuant ainsi l'idée d'un tout; les pièces éliminées feront partie des Bunte Blätter, op. $99\left(n^{\circ} 6\right)$ et des Albumblätter, op. 124, $\mathrm{n}^{0} 4$ (« Walzer »), $\mathrm{n}^{0} 11$ (« Romanze »,) $\mathrm{n}^{0} 15$ (« Walzer »), $\mathrm{n}^{\circ} 17$ («Elfe »). Le 2 juin 1837, Schumann a « fini avec le Carnaval ${ }^{20}$, mais l'œuvre ne paraîtra qu'en septembre 1837 avec son titre français : CARNAVAL / Scènes mignonnes / composées / pour le Pianoforte / sur quatre notes / et dédiées / A Mons ${ }^{r}$ Charles Lipinski / par / Robert Schumann $^{21}$.

Le tableau 1 (voir à la fin de l'article) présente la liste des pièces du Carnaval, les indications de tempo, le soggetto cavato $^{22}$ utilisé comme idée

17 « [...] - am Karnaval fortgefahren der schon December 1834 in Zwickau begonnen war» Schumann, Tagebücher, Band I : 1827-1838, 421.

18Luebbe, A History of Robert Schumann, 105 n. 102.

19 « Ich nehme hier die Gelengheit wahr, Sie, wie ich schon längst im Sinne hatte, um Verlagsübernahme zweier Compositionen von mir zu ersuchen. Eine heißt: "Carnaval"; die andere : "Phantasien [Phantasiestücke] für Pianoforte." Der Carnaval erscheint gleichzeitig, aber in etwas anderer Gestalt und mehr für die Franzosen verändert, bei Schlesinger in Paris, und würde ich Sie bitten, dessen Firma mit auf dem Titel zu erwähnen. » Jansen, Robert Schumanns Briefe, 421.

20 « Erledigt. Mit dem Carnaval. Ob sie bis letzten Juli damit fertig sein könnten. / Vom Honorar nichts gesagt. » Schumann, Briefverzeichnis $n^{\circ}$ 224, dans Luebbe, A History of Robert Schumann, 110.

21 Ibid., 111-12.

22 « Soggetto cavato est un terme italien qui désigne un motif musical façonné à partir des mots. Il se définit comme un sujet musical ou cantus firmus dérivé d'un texte par la solmisation des syllabes dont les voyelles correspondent aux voyelles du texte "; Don Michael Randel, The New Harvard Dictionary of Music (Cambridge, Mass., et Londres : The Belknap Press of Harvard University Press, 1986), 758. Compte tenu de la fídélité de Schumann à l'égard de la musique de la Renaissance, en 
thématique, soit sous sa forme originale à quatre notes ( $\mathrm{ASCH})$ ou à trois notes $(\mathrm{AsCH})$. La dernière colonne indique la tonalité du morceau.

\section{Des lettres et des notes}

L'idée de composer le Carnaval remonte à 1834, au moment où Schumann, alors étudiant de Friedrich Wieck, se prend d'affection pour une étudiante de celui-ci, Ernestine von Fricken (1816-44), qui réside chez son professeur pour ses leçons de piano. Ernestine est originaire de Asch, petit village de Bohême près de Leipzig, dont les lettres constitueront l'idée motivique à la base de la construction du cycle.

Je viens de me rendre compte que Asch est un nom très musical, que les mêmes lettres se retrouvent dans mon nom, et [qu'elles] sont les seules musicales ${ }^{23}$.

L'union des mots et de la musique est une caractéristique fondamentale chez Schumann ${ }^{24}$. Elle trouve sa manifestation dans les œuvres vocales et instrumentales, comme le montrent les titres de sa musique pour piano seul. Non seulement Schumann réconcilie-t-il ainsi deux penchants marqués de sa personnalité - la littérature, vers laquelle il a toujours été attiré et dont il deviendra l'un des ardents défenseurs et praticiens, et la musique, qui sera sa carrière - , il souscrit en plus aux canons esthétiques prédominants à son époque selon lesquels l'adjonction d'un référent extramusical confère une validité à la signification même de l'œuvre musicale, courant auquel Berlioz, Liszt et Wagner ont adhéré. Comme l'a fait remarquer Dahlhaus :

Berlioz se sentait obligé d'étendre l'appareil orchestral précisément parce qu'il sentait, secrètement, que la musique instrumentale manquait de « substance » : on imposerait une signification à la musique en multipliant ses moyens - par la rhétorique ${ }^{25}$.

particulier de celle de Palestrina, je garderai ce terme, car il répond bien aux valeurs esthétiques du romantisme. Les anagrammes musicaux en tant que source d'inspiration étaient aussi pratiqués par les compositeurs de la Renaissance et du Baroque, Bach en ayant fait grand usage.

23 « Denn eben habe ich herausgebracht, daß Asch ein sehr musikalischer Stadtname ist, daß dieselben Buchstaben in meinem Namen liegen, und gerade die einzigen musikalischen drinnen sind. » Hermann Erler, Robert Schumann's Leben, aus seinen Briefen geschilder, 2 vol. (Berlin : Ries und Erler, 1886), 1:57.

24Les principaux articles et ouvrages traitant du lien entre musique et mots dans les auvres de Schumann sont les suivants : Edward A. Lippman, «Theory and Practice in Schumann's Aesthetics », Journal of the American Musicological Society 17, $\mathrm{n}^{0} 1$ (printemps 1964) : 310-45; Eric Sams, " A Schumann Primer? », The Musical Times 111, $\mathrm{n}^{\circ} 1533$ (novembre 1970) : 1096-97; "Schumann and the Tonal Analogue », dans Schumann : The Man and His World, édit. par Alan Walker (Londres : Barry and Jenkins, 1972); «Why Florestan and Eusebius? », The Musical Times 108, $\mathrm{n}^{\circ} 1488$ (février 1967): 131-34. Anthony Newcomb, "Once More "Between Absolute and Program Music" : Schumann's Second Symphony ", 19th-Century Music 7, $\mathrm{n}^{\circ} 3$ (avril 1984) : 233-50; Thomas Alan Brown, The Aesthetics of Robert Schumann.

25 « Berlioz felt compelled to expand the orchestral apparatus precisely because he secretly sensed that instrumental music lacked "substantiation" : meaning was to be forced upon music by multiplying its devices - by rhetoric. » Carl Dahlhaus, Nineteenth-Century Music (Berkeley, Calif. : University of California Press, 1989), 250. 
Le soggetto cavato figure parmi les procédés musicaux ressucités au début du XIX ${ }^{\mathrm{e}}$ siècle permettant le pont entre la musique et le mot. D'une certaine manière, il répond à un mysticisme voulant que les lettres soient un terreau symbolique fertile pour l'inspiration des compositeurs. Ainsi, des noms deviennent des motifs musicaux et ABEGG, GADE, BACH et ASCH ont autant de résonance musicale que symbolique. Musicalement, le motif $\mathrm{ASCH}$, né des lettres, unifie structuralement le tout et, symboliquement, renvoie à des personnages. ASCH donne donc lieu à une lecture double et devient un référent musical qui, en s'adjoignant à la musique, «transmet l'idée de quelque chose (que cette chose soit présente ou non) ${ }^{26}$, ici la source de l'inspiration et la construction de l'œuvre musicale. Plus encore, il constitue une donnée fondamentale du style de Schumann au cours des années 1830-40, soit la caractérisation musicale de personnages. Brown fait remarquer que le jeune Schumann amusait ses amis en les caractérisant par des improvisations au piano et en leur demandant de deviner qui était ainsi représenté 27 .

\section{Le Carnaval et ses titres}

Le tableau 2 (voir à la fin de l'article) récapitule les titres du Carnaval et de chacun de ses mouvements tels qu'ils figurent sur les esquisses, la première édition (Erstdruck) et l'exemplaire que Schumann possédait des premières éditions de ses œuvres et désigné tout simplement par Handexemplar (exemplaire personnel). Dans la première colonne se trouvent les titres du cycle et de chaque mouvement et, à l'extrême droite du tableau, quelques remarques complémentaires. Le tableau commence par la liste des manuscrits autographes et de leur emplacement, puis figurent les données de la page de titre et de la page de garde (Vorsatzblatt) de l'exemplaire personnel. Des erreurs orthographiques apparaissent sur les esquisses et sont de la main de Schumann; par souci de fidélité aux manuscrits, elles ont été reproduites ici. Enfin, «Wsk » renvoie aux cahiers d'esquisses Wiede (Wiede Skizzenbücher, Wsk), où le numéro du cahier est suivi du numéro de la page (Wsk 1:104 signifie premier cahier, page 104), et AT, aux archives d'Alice Tully, anciennement collection Vietinghoff.

Peu d'esquisses ont survécu, les seules étant reliées au " Préambule », à « Pierrot », à « Arlequin », à « Eusebius » et à « Papillons », ou à des pièces n'ayant pas été intégrées a posteriori au Carnaval. Le titre et certains intertitres ${ }^{28}$ des esquisses ont été modifiés dans la première édition, tandis que sept

26 «A symbol conveys the idea of something (whether it is present or not). » Plantinga, Schumann as Critic, 122.

27 Brown, The Aesthetics of Robert Schumann, 164.

28 C'est Gérard Genette qui utilise le terme «intertitre » pour désigner « le titre d'une section de livre : parties, chapitres, paragraphes d'un texte unitaire, ou poèmes, nouvelles, essais constitutifs d'un recueil. » En musique, les pièces d'un cycle sont analogues aux parties constitutives du tout littéraire en ce que les miniatures s'insèrent dans un ensemble. D'une manière générale, les miniatures d'un cycle sont unies par des paramètres tels le fragment mélodique, l'harmonie, le rythme, et créent avec le tout un lien architectonique, comme les chapitres ou parties d'un roman. Pour cette raison, il me semble approprié d'adopter le même terme pour désigner les parties constitutives du cycle musical. Pour en savoir davantage sur les intertitres et leur fonction, voir Genette, Seuils, 272. 
autres pièces ou fragments ont été soit rejetés (« Capriccio », « Fantasie sopra un Tema di quatre suoni »), soit récupérés dans d'autres œuvres (op. 99, $\mathrm{n}^{\circ} 6$; op. $124, n^{\text {os }} 4,11,15$ et 17) ${ }^{29}$. Il est clair que Schumann avait l'intention d'écrire une série de variations à partir des Sehnsuchtswalzer, D. 365, de Schubert, comme le montrent les intertitres, mais ces derniers ne permettent pas de savoir si Schumann concevait l'ensemble comme un tout cohérent bâti à partir de ce thème. Tout au plus, les données préliminaires d'intitulé nous fournissent-elles la source d'inspiration, le genre (valse) et une piste quant au caractère des pièces. Il s'agit d'une série de danses, genre dominant chez Schubert, le jeune Schumann ${ }^{30}$, Chopin et bien d'autres romantiques de cette époque.

Dans la première édition du Carnaval, le titre qui renvoie au thème de Schubert est remplacé par celui de "Préambule ». En réalité, seules les mesures 8 à 10 du thème de Schubert seront conservées par Schumann; elles correspondent aux mesures 7 à 10 du Carnaval.

L'étude comparée des variantes du titre et des intertitres soulève des interrogations sur le processus de composition de Schumann et permet déjà de constater que des fragments sont réutilisés ou récupérés au profit d'autres œuvres et les titres, s'ils changent en nomenclature, devront préserver leur nature originelle, c'est-à-dire l'esprit de la pièce.

\section{Processus de composition et d'intitulation}

Le processus d'intitulation du Carnaval n'est pas différent de celui de cycles tels Papillons, op. 2, Davidsbündlertänze, op. 6, et Kreisleriana, op. 16: les idées musicales naissent de Stimmungen, d'événements, de situations, et les intitulés qui s'associent à l'œuvre musicale infléchissent la compréhension de cette œuvre. L'étude des esquisses et des premières éditions révèle un processus compositionnel en gestation, c'est-à-dire une démarche créatrice ininterrompue, en perpétuel devenir, depuis l'émergence de l'idée jusqu'à sa réalisation musicale finale (la publication de l'œuvre). Dans le Carnaval, Schumann procède par interchangeabilité des fragments musicaux : certaines pièces feront partie du cycle dans sa forme finale (« Sehnsuchtswalzervariationen », « Notturno », « Burla », « Thema », « Ecossaise »); d'autres seront mises de côté pour être utilisées plus tard dans des œuvres différentes (« Fantasia sopra un Tema di quatre suoni », «Ziemlich langsam », « Ballo », « Ro-

29 Seule la $15^{\mathrm{e}}$ pièce de l'op. $124 \mathrm{n}$ 'est pas construite sur le motif ASCH; toutes les autres le sont et leurs tonalités sont proches de celles du Carnaval : op. $99, \mathrm{n}^{0} 6$ (la b op. 124, $\mathrm{n}^{0} 4$ (la mineur), $\mathrm{n}^{0} 11$ $s i b), \mathrm{n}^{\circ} 15$ (la mineur), $\mathrm{n}^{\circ} 17(\mathrm{l} a \mathrm{~b}$ ). Luebbe présume que ces pièces auraient été composées en 1833 et en 1834 .

30Marie-Luise Maintz, Franz Schubert in der Rezeption Robert Schumanns : Studien zur Ästhetik und Instrumentalmusik (Kassel : Bärenreiter, 1995). Dans sa thèse, Maintz démontre l'influence de la musique de danse de Schubert sur Schumann. À travers divers exemples puisés dans Papillons, op. 2 $\left(\mathrm{n}^{\mathrm{os}} 8\right.$ et 11), Davidsbündlertänze, op. 6, et Carnaval, op. 9 (esquisses portant le titre Walzer, Valse noble, Valse allemande et, enfin, l'inclusion de la Großvatertanz), elle va jusqu'à conclure que le vocabulaire musical de Schumann s'est enrichi considérablement à partir de la musique de danse de Schubert. Sa démonstration est particulièrement éloquente lorsqu'elle compare la deuxième d'un groupe de 16 Allemandes et 2 Écossaises (D. 783) de Schubert avec la troisième pièce ("Arlequin ») du Carnaval de Schumann, où le rythme et le caractère sont étonnamment similaires (p. 298-99). 

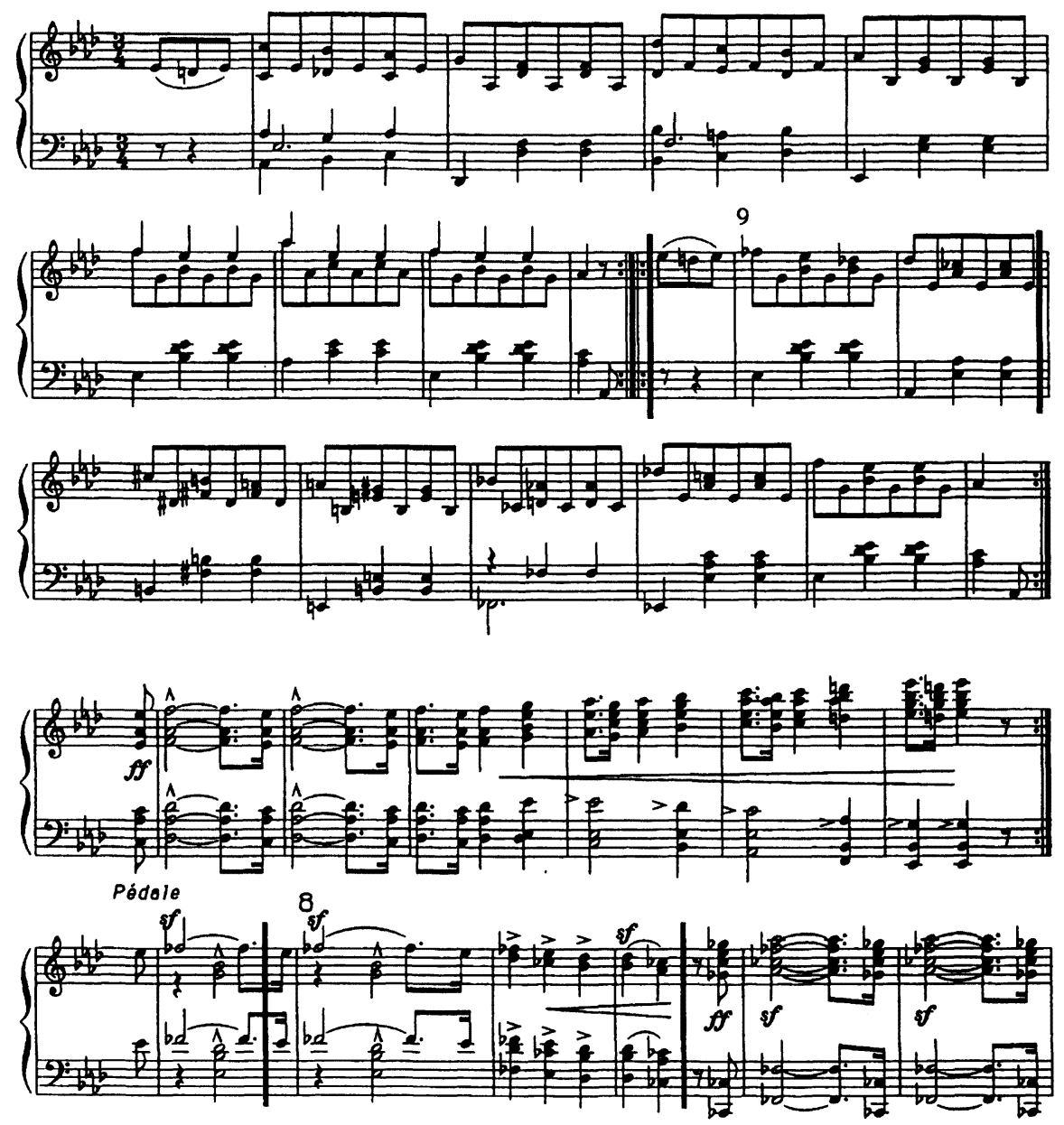

Exemple 1 : Franz Schubert, « Trauerwalzer », extrait des Sehnsuchtswalzer, D. 365 (mes. 8-10); Robert Schumann, « Préambule », extrait de Carnaval, op. 9 (mes. 7-10)

manze », "Walzer », «Elfe »). Schumann se constitue en quelque sorte une banque d'idées musicales répondant à des Stimmungen aussi variées que nombreuses. Or, les transformations apportées au titre et aux intertitres montrent que le processus d'intitulation est similaire, que cette interchangeabilité des fragments musicaux trouve son analogon dans le processus d'intitulation, car les mots peuvent être substitués, remplacés, modifiés et supprimés. En ce sens, les deux processus reflètent un même mode créateur qui semble servir une même esthétique référentialiste : le renvoi à des Stimmungen. Quant à savoir si ces processus opèrent en synchronie, Schumann affirme que les titres sont généralement apposés après la réalisation musicale. 
Un morceau fut achevé après l'autre, et le tout fut prêt pour le carnaval de 1835, mais bien dans une disposition d'esprit sérieuse et des rapports caractéristiques. Plus tard, je donnai des titres aux différents morceaux et j'appelai la collection Carnaval. Bien qu'il puisse y avoir là plus d'une chose qui charme tel ou tel auditeur, toutefois les pensées musicales se transforment trop vivement pour que tout un public puisse suivre, qui ne veut pas ainsi être effarouché toutes les minutes ${ }^{31}$.

Je doute toutefois que les titres « Chopin » et « Paganini » aient été apposés après la composition. Le style musical de ces pièces reproduit les spécificités stylistiques de chaque compositeur : les arpèges et la grande ligne mélodique chez Chopin; la virtuosité, les sauts en octaves et la vélocité de Paganini. Ici les résonances (sonores) de l'accord de septième de $m i b$, tout juste avant de reprendre la valse (mes. 36-37), sont-elles des allusions musicales au diable du violon? Ou encore des effets sonores diaboliques? Il n'existe malheureusement aucune donnée poïétique à l'endroit de ces pièces sinon que "Chapeau bas, messieurs, un génie! "32 (à propos de Chopin), et que l'impression laissée par Paganini lors d'un concert à Francfort aurait été l'élément déclencheur de la carrière musicale de Schumann. Il serait curieux et improbable que Schumann ait composé la musique sans même penser à l'un ou l'autre des musiciens. C'est sans doute un cas d'intitulation où l'idée musicale est tributaire de l'extramusical et où l'intitulé doit mener explicitement aux intentions du compositeur. L'intitulé revêt alors une fonction nouvelle : il devient germe stylistique et sa présence en regard de l'œuvre est essentielle pour la valeur ou la «signification » de la pièce. Cela met en lumière l'ambiguïté qui peut exister dans le processus d'intitulation en général chez Schumann et celui propre au Carnaval en particulier. Dans les cas où l'extramusical aurait pu précéder la musique, la fonction référentialiste de l'appareil titulaire se trouve renforcée.

\section{« Carnaval », français pour Fasching}

Le titre « Carnaval » est le fruit d'une coïncidence entre un événement et la fin de la composition musicale. Cet événement, le carnaval ou Carnelevare ${ }^{33}$ (chair, adieu) précédant le carême, a une importance considérable dans la société allemande en général et est significative chez Schumann en particulier.

31 « Ein Stück ward nach dem andern fertig und dies gerade zur Karnavalszeit 1835, überdies in ernster Stimmung und eigenen Berhältnissen. Den Stücken gab ich später Überschriften und nannte die Sammlung "Karnaval". Mag manches darin den und jenen reizen, so wechseln doch auch die musikalischen Stimmungen zu rasch, als daß ein ganzes Publikum folgen könnte, das nicht alle Minuten ausgescheucht sein will. » Schumann, « 87. Franz Liszt. II. », dans Gesammelte Schriften, 1:484 (traduction française de Rémy Stricker, Robert Schumann, le musicien et la folie [Paris : Gallimard, 1984], 49).

32Schumann, Gesammelte Schriften, 1:5.

33 « Carnelevare [...] provient de la fusion de carne (viande) et de levare (lever). Le mot carnaval, qui est carnelevare en napolitain et carnevale en toscan, veut dire que c'est le moment où l'on a "levé" les mains loin des aliments carnés et où on leur a dit adieu, vale. Le goût de se revancher parodiquement des choses sacrées se comprend chez les Chrétiens, dont la religion était obligatoire, alors que les cultes païens ne l'ont jamais été. » Pier Giovani D'Ayala et Martine Boiteux, Carnavals et mascarades (Paris : Bordas, 1988), 42. 
Ce thème ponctue la trajectoire musicale et personnelle de Schumann et prend des apparences tantôt musicales, tantôt symboliques.

L'histoire du carnaval est celle de « la fête en absolu qui concentre les divers signes du festif. [...] Durant un moment de l'année, il est permis, et même suggéré, d'être "autre" [...] Les personnifications insolites se multiplient : bossus, contrefaits et masques doubles mi-homme mi-femme $[\ldots]^{34}$. » La place du carnaval dans l'histoire culturelle allemande est légendaire. C'est le lieu de la danse, des bals masqués, du laisser-aller et de la frivolité. En renvoyant à un fait aussi connu que vécu, l'intitulé éveille la sensibilité de l'auditeur à la réalité sociologique du carnaval et de ses bals masqués, tandis que les intertitres valident ce renvoi : la composition musicale de Schumann réunit les personnages allégoriques de la commedia dell'arte («Pierrot », « Arlequin », « Pantalon et Colombine »), des personnages réels mais à la désignation relevant du masque (« Eusebius » [Schumann], "Florestan » [Schumann], "Chiarina » [Clara Wieck] et «Estrella » [Ernestine von Fricken]), des personnages réels, sans masque ( "Chopin » et " Paganini »), et des situations propres à la fête (« Valse noble » et « Valse allemande », «Aveu », « Promenade », « Reconnaissance » (« l'un des jeux favoris des bals masqués » ${ }^{35}$ ) et « Marche des "Davidsbündler" contre les Philistins »).

Le titre français attire l'attention sur deux éléments : le contexte historique dans lequel évoluait Schumann et la pratique éditoriale liée à la musique. Le premier renvoie au fait que la langue intellectuelle et littéraire de l'Allemagne, au début du XIX ${ }^{\mathrm{e}}$ siècle, était encore le français. Cette donnée historique fournit l'explication d'une pratique d'intitulation propre aux romantiques allemands : les très nombreux intitulés musicaux français des compositions de Beethoven, de Schubert et de Schumann, entre autres. Dans son sens plus large, l'emploi du français n'est-il pas une autre réalité des relations historiques entre Franca et Germania? En d'autres mots, l'histoire à laquelle faisait allusion le titre Carnaval pourrait tout aussi bien être la subtile allusion à l'Histoire.

Le deuxième élément, à mon avis plus convaincant, relève de la praxis éditoriale : Schlesinger, l'éditeur français du Carnaval, voulait un titre plus approprié et plus séduisant pour le public parisien que le titre original allemand Fasching : Schwänke auf 4 Noten. Or, Fasching se traduit en français par " carnaval ». Ces relations compositeur-éditeur indiquent que l'art d'intituler est soumis à certaines contraintes : le choix d'un titre est tributaire de facteurs commerciaux et économiques qui ont une incidence directe sur sa nature. Schumann a modifié le titre de l'œuvre pour la rendre plus « vendable ». Ceci n'est en soi pas unique dans l'histoire de la musique, mais il mérite tout de même d'être mentionné pour éviter des conclusions erronées quant à la signification du titre d'une œuvre. 


\section{Scènes mignonnes}

Le sous-titre en rubrique renseigne sur deux points : un genre musical et une pratique de traduction. Jacques Chailley décrit les scènes mignonnes comme « des miniatures comportant un nombre limité d'idées thématiques, 1 ou 2 tout au plus, et qui évoquent une idée ou un climat ${ }^{36}$. » $\mathrm{Ce}$ sont bien des miniatures qui composent la matière première du Carnaval et Schumann s'est particulièrement bien illustré dans ce genre, à en juger par ses nombreuses pièces pour le piano et ses lieder. La miniature, typique à son époque et cultivée par nombre de compositeurs, se suffit à elle-même; elle englobe avec elle une finalité, circonscrit un contenu et condense le propos. L'expression « scènes mignonnes » propose donc des tableautins, une sorte de "théâtre » exprimé en musique, validant du même coup l'esprit de la fête propre au carnaval et justifiant, une fois de plus, la présence des personnages de la commedia dell'arte. Du point de vue musical, le Carnaval est une constellation de fragments pouvant être considérés comme auto-suffisants, à l'exception de quelques pièces qui s'enchaînent. La musique rend donc ce que le sous-titre annonce.

La traduction du sous-titre soulève un problème sémantique. "Schwänke » signifie littéralement facéties, farces, bouffonneries ou histoires drôles, et est à mon sens plus précis que le français « scènes mignonnes ». L'allemand saisit mieux l'intention musicale du compositeur et valide le choix de pièces caractéristiques comme genre. L'expression "scènes mignonnes » affaiblit le caractère évoqué par l'allemand. $\mathrm{Si}$ "scène » est défini comme un « événement qui offre une unité, présente une action, constitue un spectacle remarquable ou éveille des sentiments » (Robert), son complément " mignonne » semble affaiblir le caractère inféré par l'allemand. La traduction française introduit une certaine dissonance sémantique, née de l'imprécision des mots choisis par Schlesinger et à distance de la réalité compositionnelle de Schumann. Le français est déductif en comparaison avec l'allemand (comme l'anglais d'ailleurs), plus descriptif ${ }^{37}$. En revanche, l'ambiguïté du français accroît le potentiel symbolique du titre et son aire sémantique, elle propose des avenues moins circonscrites pour l'auditeur. Cela pose certes le problème de la fidélité à l'intention du compositeur, mais on peut présumer que Schumann connaissait suffisamment le français pour sanctionner la traduction de Schlesinger.

\section{Sur quatre notes : ASCH}

$\mathrm{ASCH}$, dans le sous-titre, renvoie au procédé du soggetto cavato mentionné plus haut et dont les premières traces sont décelables bien avant la composition du Carnaval. Cette pratique, chez Schumann, remonte à la composition du

36 Jacques Chailley, Carnaval de Schumann (op. 9) (Paris : Alphonse Leduc, 1971), 7.

37En anglais, une phrase comme « don't move your head, your body, your arms, your legs... » se traduirait en français par « reste immobile ». Or, ce serait dénaturer l'esprit du français que de procéder, comme l'anglais, de manière descriptive plutôt que déductive. L'allemand se rapproche beaucoup de l'anglais, comme le révèlent les exemples suivants : Krankenhaus (hôpital) = maison des malades; Handschuhe (gants) = souliers pour les mains. On peut multiplier les exemples du genre. 
Thème sur le nom Abegg varié pour le pianoforte (1830, publ. 1831), et il y aura recours jusqu'à la fin de sa vie, comme dans les Six fugues sur le nom $B A C H(1845)$ et la Sonate pour violon et piano F.A.E. (1853), qui fait référence au motto de Joseph Joachim, «Frei aber einsam ». Outre le fait que le soggetto cavato apparaisse comme la réconciliation symbolique entre deux penchants, le caractère ludique et mystificateur des codes secrets y est sans doute pour quelque chose dans l'attirance de Schumann pour cette pratique.

\section{Jeu de notes}

Il reste à préciser un dernier élément en ce qui concerne le soggetto cavato ASCH : les lettres constitutives donnent lieu à des possibilités musicales liées à l'altération (bémol/dièse) des notes. En allemand, les altérations sont des suffixes que l'on appose aux notes : "es " indique les bémols et "is » les dièses. Ainsi, la b s'écrit « Aes », mi b «Ees », do ฤ « Cis » et ainsi de suite. Cependant, pour des raisons d'euphonie, certaines désignations de notes peuvent être modifiées et devenir équivoques pour ce qui est de la note véritablement désignée. C'est le cas pour la lettre $S$ de Asch qui engendre deux possibilités musicales. D' abord, elle peut être considérée comme suffixe de $A$, auquel cas elle est écrite en minuscules et résulte de la contraction de «Aes »; les Allemands écrivent «As» (soit lab du «Sphinx 2 »). Mais $S$ est aussi le résultat d'une contraction en deux temps ou d'une double contraction : "Ees » (pour $m i$ b) qui, pour des raisons d'euphonie, s'écrira « Es » et se prononcera $S$ («Sphinx 3 »), qu'il s'agisse de «Ees » ou de «Es». Schumann joue ici avec le potentiel euphonique d'une lettre, qui lui-même est porteur de deux possibilités musicales. Au jeu des lettres correspond un jeu de notes.

Si Schumann était conscient des jeux de lettres possibles dans un nom et de leur potentiel musical, on peut présumer - et il n'est question ici que de cela - qu'il était sensible aussi à un certain « pouvoir » musical lié à la prononciation des lettres. Les trois idées motiviques nées de la transformation des lettres et de leurs possibilités musicales sont les suivantes :

«Sphinx 1 »: SCHA (mi b, do, si, la)

«Sphinx $2 »:$ AsCH (la b, do, si)

«Sphinx 3 »: ASCH (la, mi b, do, si)

Sphinx 1
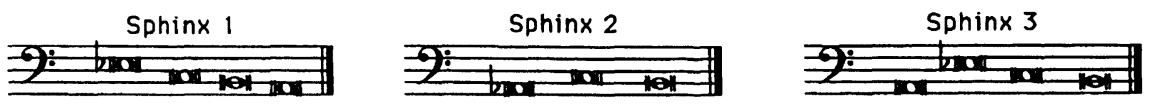

Exemple 2 : « Sphinx »

Pour ce qui est des intertitres de certaines pièces du cycle, Schumann a laissé quelques explications qui sont reproduites ci-dessous ${ }^{38}$.

38Tiré de Jansen, Schumanns Briefe, 101-2. À moins d'indication contraire, les traductions sont de l'auteur. 
"ASCH-SCHA (lettres dansantes) » : ville natale d'Ernestine von Fricken, inspiratrice de l'œuvre; les lettres correspondantes se retrouvant dans le nom du compositeur.

«Chiarina » : portrait de Clara Wieck.

« Estrella » : nom comme on en met sous un portrait pour mieux en fixer le souvenir ${ }^{39}$.

« Reconnaissance »: scène de reconnaissance.

«Paganini »: violoniste et compositeur dont le concert donné à Francfort a été décisif dans le choix d'une carrière musicale pour Schumann.

«Aveu » : aveu d'amour.

« Promenade » : une promenade comme il s'en prend lors de bals allemands bras dessus, bras dessous avec une compagne.

\section{Le tout et ses parties}

Le motif mélodique (voir le tableau 1) se trouve au début des pièces, presque exclusivement sous forme mélodique, sauf pour "Valse noble ». Sept des pièces sont basées sur le motif ASCH («Sphinx $3 »)$ tandis que 12 le présentent sous sa forme AsCH («Sphinx $2 »)$; curieusement, SCHA ne s'y retrouve pas. En excluant les «Sphinx » (ou «Sphinxes » comme l'écrit Schumann), Carnaval se compose de 21 pièces fondées sur les deuxième ou troisième des «Sphinx », sauf deux exceptions : « Réplique » et « Chopin », qui en sont donc exempts.

L'originalité du cycle tient à l'innovation que Schumann apporte au principe du thème et variations : le cycle n'est ici pas articulé selon la transformation de la mélodie, mais sur un fragment motivique en tant que lien structurant du tout. Schumann se permet une facture autre, justifiant du même coup une intitulation autre, voire plus fantaisiste. En outre, il est frappant que le motif issu des possibilités euphoniques des lettres soit prédominant par rapport au motif original, c'est-à-dire celui mettant en musique le nom $\mathrm{ASCH}$, sans altération. Est-ce là une autre spécificité de la pensée schumannienne, celle liée au jeu? Il est possible de le croire, compte tenu du caractère ludique inhérent au cycle.

La forme macroscopique du cycle (exemple 3) est de type $A B \mid B^{\prime} A^{\prime}$, ce qui laisse transparaître une symétrie fondée sur le principe du cycle : " une œuvre qui se clôt avec une reprise variée ou élaborée de la pièce d'ouverture ${ }^{40}$. En reprenant de larges fragments du « Préambule » (mes. 71-88 et 115-33) dans la « Marche» (mes. 83-100 et 225-43), Schumann donne une forme cyclique qui autorise à désigner ces deux pièces par $\mathrm{A}$ et $\mathrm{A}^{\prime}$ respectivement. Entre ces

39 Traduction de Stricker, Schumann, 49.

40 « They are literally cyclic, in that they close with a varied and/or extended reprise of the opening piece. » Peter Kaminsky, « Aspects of Harmony, Rhythm and Form in Schumann's Papillons, Carnaval and Davidsbündlertänze » (thèse de doctorat, Eastman School of Music, 1989), 1:137. 
deux pôles, les pièces 2 à 20 forment une large partie centrale faite de deux pans qui sont en rapport de miroir : la première partie opère macroscopiquement sur deux quintes ascendantes $(L a b-M i b-S i b)$ et la deuxième partie lui répond, à la fois symétriquement et irrégulièrement, à travers une progression de quinte descendante $(M i b-L a b)$. Si l'on tient pour principe qu'une symétrie est possible lorsqu'il existe une "similitude des deux moitiés d'une chose » (Robert), l'organisation similaire des deux pans et l'homologie de structure entre l'harmonie et le motif utilisé rendent le Carnaval symétrique. Il faut toutefois relever l'asymétrie : le nombre de pièces est inégal dans les deux parties ( 9 contre 12) et le parcours tonal couvre un ambitus différent dans l'une et l'autre partie (deux quintes contre une).

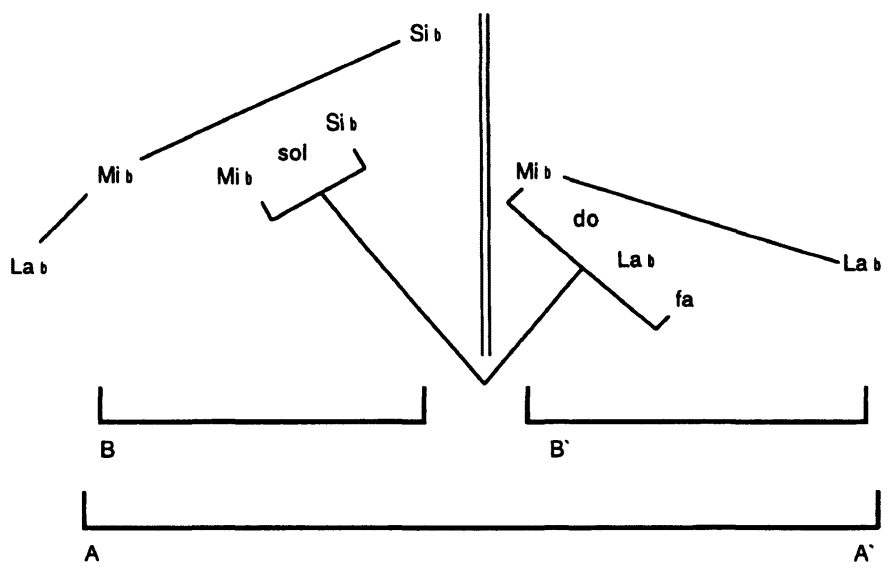

Exemple 3 : Symétrie et asymétrie de la forme globale du Carnaval

Il me semble percevoir un autre jeu qui corroborerait cette idée de symétrie, mais cette fois à l'échelle microscopique : certaines pièces se jumellent pour former un couple, deux entités qui se complètent, deux opposés qui s'annulent ou trouvent leur sens dans le rapport que ces pièces entretiennent, un rapport à la fois musical et symbolique :

1. «Eusebius » et «Florestan » sont les opposés qui se complètent, les deux antipodes d'une même réalité, le premier rêveur (adagio), le second fougueux (passionato);

2. «Pierrot »- « Arlequin » et « Coquette »- « Réplique » reprennent un fragment mélodique similaire dans l'un et l'autre couple : le motif descendant (mib-do-sib) de la mesure 3 de «Pierrot " semble être reproduit en augmentation dans « Arlequin " à ceci près d'une note ajoutée, et l'introduction de "Coquette » se retrouve dans « Réplique ». 
3. «Chiarina » (passionato) et «Estrella ( boliquement : la première lettre du nom invite à une double allusion, $C$ pour Chiarina/Clara, $E$ pour Estrella/Ernestine.

4. «Chopin » et «Paganini » sont deux musiciens que Schumann admirait et dont il reproduit le style musical dans les pièces éponymes.

5. Deux valses («Valse noble » et «Valse allemande ») ${ }^{41}$ dont les intitulés évoquent Schubert.

6. Et, pour finir la liste et ajouter à la mystification, «Pantalon et Colombine », personnages de la commedia dell'arte, réunis dans une même pièce.

Ainsi, ces rapports de symétrie et de jumelage à l'échelle macroscopique et microscopique valident la grande cohérence du cycle et renchérissent l'homologie de structure entre les deux niveaux.

\section{Le titre, élément symbolique}

Le titre peut être considéré en tant que symbole dans la mesure où il fait intervenir un « ensemble de relations et d'interprétations qui lui sont afférentes ${ }^{42}$ ou qu'il " transmet l'idée de quelque chose (que cette chose soit présente ou non) ${ }^{43}$. » Dès lors, le masque peut être le symbole du carnaval, lui-même symbole d'illusion et d'aspiration, comme l'indique Marcel Brion dans son essai sur Schumann.

Ce thème du bal masqué, mi-réel, mi-fantastique, au cours duquel les êtres, alternativement, se révèlent et se dissimulent, et par leur manière même de se cacher derrière des masques, révèlent encore leur nature profonde, a exercé une curieuse fascination sur Schumann; c'est le thème romantique par excellence, puisqu'il exprime la dualité de la personne, l'incertitude dans la connaissance de soi, la hantise du [D]oppelgänger $[\ldots]^{44}$

Conscient de sa propre dualité, exprimée par Florestan et Eusebius, Schumann trouvait sans doute dans l'idée du bal masqué un exutoire idéal pour ses idées artistiques et musicales : les personnages réels côtoient les imaginaires, les caractérisations musicales se succèdent et les événements se précipitent. Le fil qui sépare le rêve et la réalité est ténu, à la limite du décelable. Réel et irréel se réclament d'une même disposition d'esprit, celle propre au bal masqué, au jeu de doubles. Le masque que portent les personnages musicaux devient la manifestation de la nature double (Doppelnatur) de l'être humain, un aspect

41 Les mesures 9 et suivantes proviennent des Valses romantiques, op. 4, de Clara Wieck. L'emprunt de fragments mélodiques provenant de ses propres compositions ou de compositions d'autres compositeurs est une pratique courante chez Schumann; à ce chapitre, voir R. Larry Todd, "On Quotation in Schumann's Music ", dans Schumann and His World (Princeton : Princeton University Press, 1994).

42 Jean Chevalier et Alain Gheerbrant, Dictionnaire des symboles (Paris : Robert Laffont, 1995), xii.

43 Plantinga, Schumann as Critic, 122.

44Marcel Brion, Schumann et l'âme romantique (Paris : Albin Michel, 1986), 142. 
dont Schumann était conscient déjà en 1831, soit trois ans avant la composition du Carnaval :

J'ai parfois le sentiment que mon humanité objective veut se séparer complètement de ma subjectivité, ou comme si je me trouvais entre mon existence et mon apparence, entre la forme et l'ombre ${ }^{45}$.

Cette confidence permet de poser une adéquation entre d'une part objectivité, existence et forme et, d'autre part, subjectivité, apparence et ombre. J'oserais ici transposer cette adéquation à l'œuvre elle-même. L' " existence » du Carnaval, c'est l'œuvre elle-même, c'est sa matière musicale. Cette matière musicale est constituée d'une suite de fragments ou de miniatures dont la forme globale est imperceptible à l'écoute. En d'autres mots, le Carnaval semble avoir une forme désarticulée, faite d'une enfilade de tableautins dont l'ordre de présentation ou d'ordonnancement (agencement) est, lui aussi, imperceptible. En considérant que l'ombre se définit par « l'apparence changeante, transitoire et trompeuse d'une réalité » (Robert), le transfert de cette notion à l'œuvre qui nous occupe permet d'établir l'équation suivante. Le Carnaval donne lieu à une réalité trompeuse : elle dissimule une forme structurée et cohérente (AB|B'A') qui prend appui sur des soggetti cavati.(ASCH). À leur tour, ces soggetti cavati sont trompeurs en ce qu'ils dissimulent un réseau motivique plus organisé qu'ils ne laissent entrevoir, un réseau organique. Les soggetti cavati ne sont alors que les traits diffus de la facture musicale. Qui plus est, ils sont l'ombre d'une autre réalité, symbolique celle-là : le renvoi à Ernestine von Fricken et la clé de la construction du cycle. Les notions d'objectivité et de subjectivité telles qu'appliquées à la composition du Carnaval peuvent être résumées de la façon suivante.

\section{OBJECTIVITÉ}

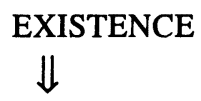

Forme<smiles>[CH]</smiles>

Miniatures

$\Downarrow$

Agencement

\section{SUBJECTIVITÉ}

APPARENCE<smiles>[CH]</smiles>

Ombre<smiles>[AlH]</smiles>

ASCH<smiles>[AlH2]</smiles>

Énigme

Le Carnaval est donc le lieu de la cohabitation de contrastes qui révèlent, pièce après pièce, une énigme dont la solution est donnée par le seul fragment inaudible du cycle : «Sphinx », symbole du monstre à l'énigme. Le titre et les

45 « Mir ist's manchmal, als wolle sich mein objectiver Mensch vom subjectiven ganz trennen oder als ständ' ich zwischen meiner Erscheinung u. meinen Syn, zwischen Gestalt und Schatten. » Schumann, Tagebücher, 1:339. 
intertitres sont alors autant de clés au jeu de masques qui s'opère dans l'œuvre musicale :

«Florestan » et « Eusebius » : Schumann

«Estrella » : Ernestine von Fricken

«Chiarina » : Clara Wieck

«Pause » : En allemand, ce mot a une double signification : arrêt et calque. Au sens d'arrêt, il symbolise l'arrêt du discours musical avant la Marche. Au sens de calque, il symbolise la reprise intégrale des mesures 87 à 114 du "Préambule ", avec la même indication de caractère.

"Marche des "Davidsbündler" contre les Philistins »: les compositeurs avant-gardistes (Davidsbündler) et les compositeurs prônant la musique de salon (Philistins) $^{46}$.

Finalement, le propos symbolique du Carnaval est magnifié par ce passage tiré des Flegeljahre de Jean Paul, passage auquel Schumann renvoie dans ses Papillons :

Un bal en masques, dit-il à sa cavalière, est peut-être la réalité la plus sublime que la vie puisse inventer à l'imitation du jeu poétique. Devant le poète, toutes les classes et tous les Temps sont à égalité ; toute apparence n'est que vêtement, l'intérieur toute joie et sons. Ainsi les hommes composent la figure poétique d'eux-mêmes et de la vie [...] la musique, pays des âmes au même titre que les masques sont le pays des corps ${ }^{47}$.

\section{Conclusion}

Le Carnaval exerce une fascination musicale et symbolique. Musicalement, il s'inscrit dans une période de regain d'intérêt pour les pièces caractéristiques, un genre saillant à cette époque du romantisme. En cela, le titre est incontournable dans notre appréhension et notre compréhension de l'œuvre musicale. Symboliquement, le titre laisse entrevoir un réseau complexe de significations et de référents, à la base même de l'esthétique schumannienne. L'appareil titulaire agit alors comme l'interface entre la réalité (la musique) et l'imaginaire (le symbolique). À travers une panoplie de masques musicaux se déploie une œuvre qui prend les allures d'un poème en puissance : le devenir poétique du Carnaval de Schumann réside, pour beaucoup, dans l'association entre l'appareil titulaire et de la musique.

46Le mot « Philistin » (de l'allemand Philister, celui qui n'est pas allé à l'université) désigne une personne vulgaire, fermée aux arts et aux lettres de même qu'à la nouveauté.

47 « Ein Ball en masque ist vielleicht das Höchste, was der spielenden Poesie das Leben nachzuspielen vermag. Wie vor dem Dichter all Stände und Zeiten gleich sind und alles Äußere nur Kleid ist, alles Innere aber Lust und Klang: so dichten hier die Menschen sich selber und das Leben nach [...] in der Musik, diesem Lande der Seelen, wie die Masken das Land der Körper sind. » Dans Jean Paul [Richter], Sämtliche Werke, Abteilung 1, Band 2, édit. par Norbert Miller (Francfort-sur-le-Main : Zweitausendeins, 1996), 1074; traduit dans Chailley, Carnaval de Schumann, 30. 
Une étude du titre de l'œuvre offre donc plusieurs perspectives d'appréhension de l'œuvre musicale: elle permet de mieux saisir certains mécanismes synchrones et asynchrones propres à l'élaboration de l'œuvre musicale et révèle une pensée créatrice multiforme qui confère à l'œuvre musicale sa densité signifiante. Comme l'écrivait Liszt :

Le Carnaval (op. 9) est une mascarade colorée d'artistes dont les groupes sont traités si directement, si énergiquement et avec éclat, qu'en reproduisant leur physionomie et en rendant leurs gestes les plus animés, le Carnaval doit figurer parmi les œuvres les plus denses et réussies de Schumann et de ce genre en général ${ }^{48}$.

48 « Carnaval (op. 9) reveals a colourful masquerade of artists whose groups are treated so directly, so energetically and vividly, that by remarkably reproducing their physiognomies and capturing their most lively gestures it must count among the richest and most successful works of the author and of this genre in general. » Franz Liszt, « Robert Schumann », dans Schumann and His World, 356. 
Tableau 1 : Liste des pièces composant le Carnaval de Schumann

\begin{tabular}{|c|c|c|c|c|}
\hline Titres & Tempo & $\begin{array}{c}\text { Soggetto } \\
\text { cavato }\end{array}$ & $\begin{array}{l}\text { Nombre } \\
\text { de notes }\end{array}$ & Tonalité \\
\hline $\begin{array}{l}\text { 1. Préambule } \\
\text { 2. Pierrot } \\
\text { 3. Arlequin } \\
\text { 4. Valse noble } \\
\text { 5. Eusebius } \\
\text { 6. Florestan } \\
\text { 7. Coquette } \\
\text { 8. Réplique } \\
\end{array}$ & $\begin{array}{l}\text { Quasi maestoso } \\
\text { Moderato } \\
\text { Vivo } \\
\text { Un poco maestoso } \\
\text { Adagio } \\
\text { Passionato } \\
\text { Vivo } \\
\text { L'istesso tempo } \\
\end{array}$ & $\begin{array}{l}\text { AsCH } \\
\text { ASCH } \\
\text { ASCH } \\
\text { ASCH } \\
\text { ASCH } \\
\text { ASCH } \\
\text { ASCH } \\
- \\
\end{array}$ & $\begin{array}{l}3 \\
4 \\
4 \\
4 \\
4 \\
4 \\
4 \\
-\end{array}$ & $\begin{array}{l}\text { Lab } \\
\text { Mib } \\
\text { Sib } \\
\text { Sib } \\
\text { Mib } \\
\text { sol } \\
\text { Sib } \\
\text { sol } \\
\end{array}$ \\
\hline \multicolumn{5}{|c|}{ Sphinx : $\mathrm{n}^{0} 1$ (SCHA), $\mathrm{n}^{0} 2$ (AsCH), $\mathrm{n}^{0} 3$ (ASCH) } \\
\hline $\begin{array}{l}\text { 9. Papillons } \\
\text { 10. ASCH-SCHA (lettres } \\
\text { dansantes) } \\
\text { 11. Chiarina } \\
\text { 12. Chopin } \\
\text { 13. Estrella } \\
\text { 14. Reconnaissance } \\
\text { 15. Pantalon et Colombine } \\
\text { 16. Valse allemande } \\
\text { 17. Intermezzo - } \\
\text { Paganini } \\
\text { 18. Aveu } \\
\text { 19. Promenade } \\
\text { 20. Pause } \\
\text { 21. Marche des } \\
\text { \& Davidsbündler * contre } \\
\text { les Philistins }\end{array}$ & $\begin{array}{l}\text { Prestissino } \\
\text { Presto } \\
\text { Passionato } \\
\text { Agitato } \\
\text { Con affetto } \\
\text { Animato } \\
\text { Presto } \\
\text { Molto vivace } \\
\text { Presto } \\
\\
\text { Passionato } \\
\text { Comodo } \\
\text { Vivo } \\
\text { Non allegro }\end{array}$ & $\begin{array}{l}\text { AsCH } \\
\text { AsCH } \\
\text { AsCH } \\
- \\
\text { AsCH } \\
\text { AsCH } \\
\text { AsCH } \\
\text { AsCH } \\
\text { AsCH } \\
\\
\text { AsCH } \\
\text { AsCH } \\
\text { AsCH } \\
\text { AsCH }\end{array}$ & $\begin{array}{l}3 \\
3 \\
3 \\
3 \\
3 \\
3 \\
3 \\
3 \\
3 \\
3 \\
3 \\
3\end{array}$ & $\begin{array}{l}\text { Mib } \\
\text { Mib } \\
\text { do } \\
\text { Lab } \\
f a \\
L a b \\
f a \\
\text { Lab } \\
f a \\
\\
\text { Lab } \\
\text { Réb } \\
\text { V/Lab } \\
\text { Lab }\end{array}$ \\
\hline
\end{tabular}


Tableau 2 : Liste des titres des pièces composant le Carnaval, op. 9 (esquisses, première édition, exemplaire personnel)

Emplacement des sources manuscrites

New York, Pierpont Morgan Library (collection Alice Tully/Vietinghoff) : 2 esquisses

Berlin, Staatsbibliothek, Stiftung Preußischer Kulturbesitz : N. Mus. Ms. $n^{\circ} 57$

Bonn, Universitätsbibliothek, Wiede Skizzenbücher : cahiers d'esquisses 1 et 2,

Mss. R. Schumann 13-17

Abréviations

AT : Collection Alice Tully

Wsk : Wiede Skizzenbücher

\begin{tabular}{|c|c|c|c|c|}
\hline Titre & Esquisse & Première édition (Erstdruck) & $\begin{array}{l}\text { Exemplaire personnel } \\
\text { (Handexemplar) }\end{array}$ & Remarques \\
\hline Page de titre & & $\begin{array}{l}\text { Carnaval / Scènes } \\
\text { mignonnes / composées } \\
\text { pour le Pianoforte / sur } \\
\text { quatre notes et / dédiés / } \\
\text { A Mons' Charles Lipinski / } \\
\text { par Robert Schumann. / } \\
\text { Propriette des Editeurs / } \\
\text { Oeuv. 9. Pr. 1 Thlr. 12 Gr. } \\
\text { / a Leipzic Chez Breitkopf } \\
\text { \& Hărtel. / a Paris chez M. } \\
\text { Schlesinger. / Enregistre } \\
\text { dans les Archives de } \\
\text { l'Union. / Lith. De Fr. } \\
\text { Krătzschmer }\end{array}$ & Idem & \\
\hline Vorsatzblatt & & Manque & & \\
\hline Preambule & $\begin{array}{l}\text {. Sehnsuchtsvariationen ", } \\
\text { Scenes musicales sur un } \\
\text { thême connu, dédies a Mad. } \\
\text { Henriette Voigt [Esquisse } \\
\text { N. Mus. ms n' } 57 \text { ] } \\
\text { Fragment, Wsk. 1:104 } \\
\text { Sariationen über } \\
\text { Sehnsuchtswalzer" (erinnert } \\
\text { an Flechsig) [fragment, } \\
\text { Wsk. 2:16, esquisse 141] }\end{array}$ & $\begin{array}{l}\text { PRÉAMBULE. Quasi } \\
\text { meastoso }\end{array}$ & $\begin{array}{l}\text { PRÉAMBULE. Quasi } \\
\text { maestoso }\end{array}$ & $\begin{array}{l}\text { Trauer-walzer, op. } 9 \text {, } \\
\text { D. 365, mes. 7-13 dans } \\
\text { Préambule = paraphrase } \\
\text { de la valse; } 4 \text { variations et } 3 \\
\text { ritournelles; esquisse } \\
\text { élaborée }\end{array}$ \\
\hline
\end{tabular}




\begin{tabular}{|c|c|c|c|c|}
\hline & $\begin{array}{l}\text { Scènes musicales sur un } \\
\text { theme connu de François } \\
\text { Schubert, comp. p. le Pfte } \\
\text { et dedies a Mad. Henriette } \\
\text { Voigt par R. Schumann / } \\
\text { Oeuv. } 10 \text { [AT] } \\
\text { Scènes mignonnes [AT] }\end{array}$ & & & $\begin{array}{l}\text { Esquisse elaborée; mes. } \\
1-24=\approx \text { Préambule "; } \\
\text { mes. 9-10= } \\
\text { "Trauermarsch» } \\
\text { Même musique que dans les } \\
\text { scènes musicales }\end{array}$ \\
\hline Pierrot & Notturno [AT] & PIERROT. Moderato & PIERROT. Moderato & \\
\hline Arlequin & Burla [AT] & ARLEQUIN. Vivo & ARLEQUIN. Vivo & \\
\hline Valse noble & & $\begin{array}{l}\text { VALSE NOBLE. Un poco } \\
\text { meastoso: }\end{array}$ & $\begin{array}{l}\text { VALSE NOBLE. Un poco } \\
\text { meastoso }\end{array}$ & \\
\hline Eusebius & $\begin{array}{l}\text { Thema [Wsk. 2:15, esquisse } \\
\text { no 137] }\end{array}$ & EUSEBIUS. Adagio & EUSEBIUS. Adagio & \\
\hline Florestan & & | FLORESTAN. Passionato & FLORESTAN. Passionato & \\
\hline Coquette & & COQUETTE. Vivo & COQUETTE. Vivo & \\
\hline Réplique & & $\begin{array}{l}\text { RÉPLIQUE. L'istesso } \\
\text { tempo }\end{array}$ & $\begin{array}{l}\text { RÉPLIQUE. L'istesso } \\
\text { tempo }\end{array}$ & \\
\hline (Sphinxes) & & SPHINXES. & SPHINXES. & \\
\hline Papillons & Ecossaise [AT] & PAPILLONS. Prestissimo & PAPILLONS. Prestissimo & \\
\hline $\begin{array}{l}\text { A.S.C.H. - S.C.H.A. } \\
\text { (Lettres dansantes) }\end{array}$ & & $\begin{array}{l}\text { A.S.C.H. - S.C.H.A. } \\
\text { Presto (LETTRES } \\
\text { DANSANTES) }\end{array}$ & $\begin{array}{l}\text { A.S.C.H. - S.C.H.A. } \\
\text { Presto (LETTRES } \\
\text { DANSANTES) } \\
\end{array}$ & \\
\hline Chiarina & & CHIARINA. Passionato & CHIARINA. Passionato & \\
\hline Chopin & & CHiOPIN. Agitato & CHOPIN. Agitato & \\
\hline Estrella & & ESTRELLA. Con affetto & ESTRELLA. Con affetto & \\
\hline Reconnaissace & & \begin{tabular}{|l}
$\begin{array}{l}\text { RECONNAISSANCE. } \\
\text { Animato }\end{array}$ \\
\end{tabular} & $\begin{array}{l}\text { RECONNAISSANCE. } \\
\text { Animato } \\
\end{array}$ & \\
\hline Pantalon et Colombine & & $\begin{array}{l}\text { PANTALON ET } \\
\text { COLOMBINE. Presto } \\
\end{array}$ & $\begin{array}{l}\text { PANTALON ET } \\
\text { COLOMBINE. Presto }\end{array}$ & \\
\hline
\end{tabular}




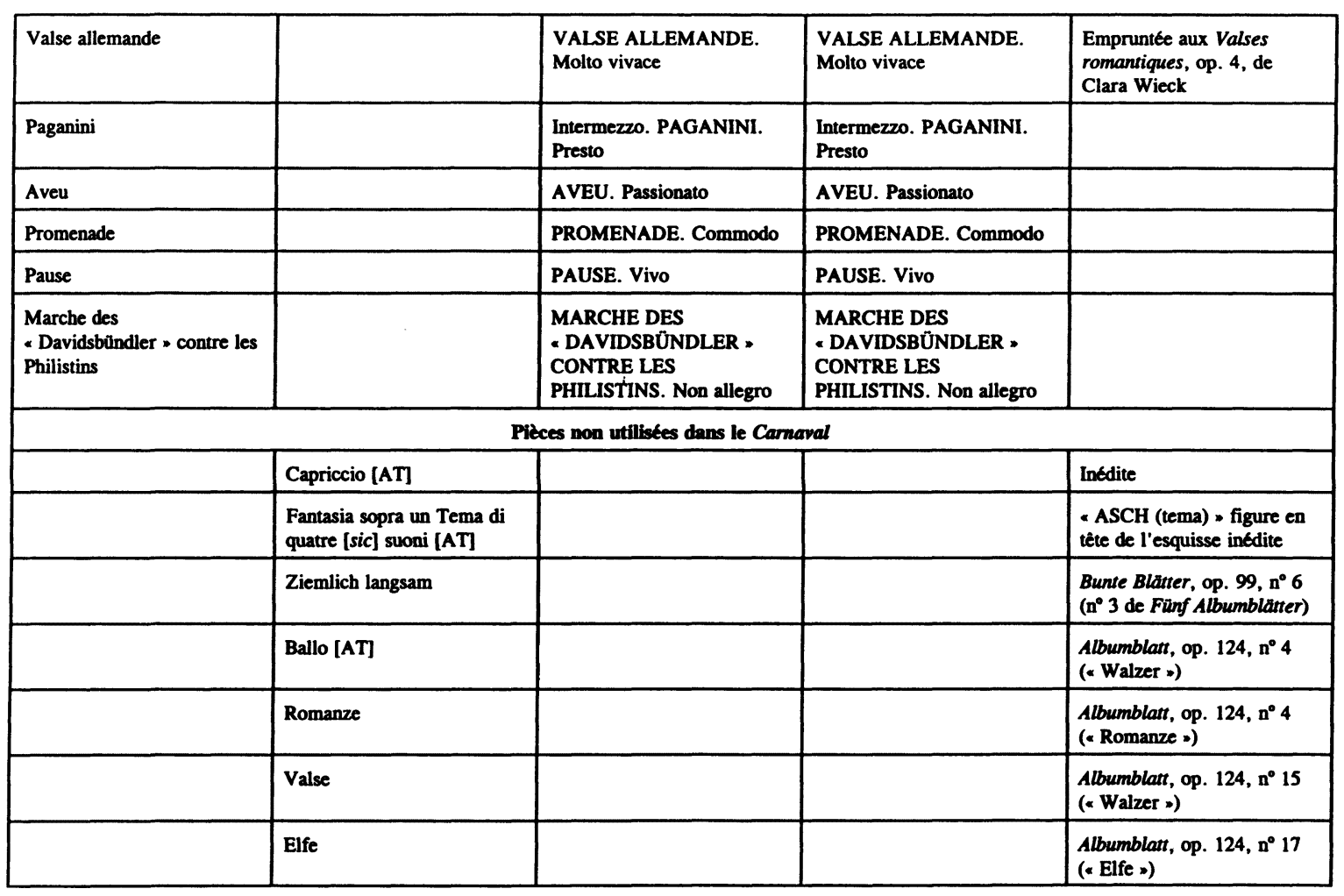




\section{Résumé}

Qu'y a-t-il dans le titre d'une œuvre musicale qui permettrait de mieux comprendre l'œuvre elle-même, sa genèse, sa construction, sa signification et le symbolisme dont elle pourrait se réclamer? Au-delà d'une constellation de fragments, le Carnaval, op. 9, de Robert Schumann porte en lui une logique motivique et symbolique que l'étude du titre permet de démasquer. L'auteur tente de montrer qu'une étude titrologique mettra au jour les liens très intimes entre musical et extramusical ainsi que le réseau de référents très complexe vers lequel le titre dirige. Le titre agit bien souvent comme interface entre l'œuvre musicale et son symbolisme. 